\title{
Cardio-Cerebral infarction syndrome: definition, diagnosis, pathophysiology, and treatment
}

\author{
Mohammed Habib* \\ Cardiology Department- Al Shifa Hospital, Gaza, Palestine
}

\begin{abstract}
Acute ischemic stroke and coronary artery disease are the major causes of death worldwide. The prevalence of coronary artery disease has been reported in one fifth of stroke patients. Although high incidence rate of acute myocardial infarction after acute ischemic stroke and the high risk of acute ischemic stroke after myocardial infarction has been reported in several studies. Any acute or recent problem in the heart or brain that could result in an acute infarction of the other. In this review we describe the definition and new classification of the cardio-cerebral infarction syndrome with 3 subtypes that reflect the pathophysiology and treatment options.
\end{abstract}

\section{Introduction}

The incidence of acute ischemic stroke (AIS) after Acute myocardial infarction (AMI) during the hospital stay ranges from $0.7 \%$ to $2.2 \%$ [1-3]. AIS occurred more frequently in the first days after AMI, but incidence progressively decreased over time [3-5]. Brandi Witt et al. [6], suggested that during hospitalization for the index myocardial infarction (MI), 11.1 ischemic strokes occurred per $1000 \mathrm{MI}$ compared with 12.2 at 30 days and 21.4 at 1 year. Positive predictors of stroke after MI included: advanced age, diabetes, hypertension, history of prior stroke, anterior location of index MI, prior MI, atrial fibrillation, heart failure, and non-white race.

The incidence of AMI after Acute ischemic stroke was relatively low and unexpectedly highest during the first year after stroke. The 5-year cumulative incidence of AMI was 2.0\%. The annual risk was highest in the first year after the index event (1.1\%), followed by a much lower annual risk in the second to fifth years (between $0.16 \%$ and $0.27 \%$. Coronary heart disease was the most substantial risk factor for AMI after stroke and conferred an approximate 5-fold greater risk [7].

Both AIS and acute ST elevation myocardial infarction (STEMI) are medical emergency conditions, which require timely diagnosis and management. The incidence of patients who diagnosed acute ischemic stroke about $0.009 \%$ [8]. In this article we divided cardio-cerebral infarction syndrome into 3 types according to AIS or AMI although diagnostic criteria, pathophysiology, and treatment options according to recent trials or case series.

\section{Objectives}

- Identify the definition and etiologies of cardio-cerebral infarction syndrome.

- Describe the pathological findings in a patient with each subtype of cardio-cerebral infarction syndrome.

- Outline the treatment and management options available for patients with each subtype of cardio-cerebral infarction syndrome.

\section{Definition of cardio-cerebral infarction syndrome}

Cardio-cerebral infarction syndrome can generally be defined as Primary disorders (infarction or its complications) of 1 of these 2 organs (Heart or Brain) often result in secondary infarction/injury to the other or to both organs (Figure 1).

\section{Types of cardio-cerebral infarction syndrome}

- Type I: concurrent cardio-cerebral infarction syndrome

- (Type IA: Cardiac causes, Type IB: brain Cause, Type IC: noncardiac and non- brain causes)

- Type 2: Acute ischemic stroke after recent myocardial infarction

- Type 3: Acute myocardial infarction after recent ischemic stroke

- The recent Ischemic stroke: Ischemic stroke in the previous 3 months

- The recent myocardial Infarction: myocardial infarction in the previous 3 months (Figure 2)

\section{Type I: concurrent cardio-cerebral infarction syndrome}

Definition: Concurrent cardio-cerebral infarction syndrome can be diagnosed by the presence of simultaneous acute onset of a focal neurological deficit, indicating acute stroke and a chest pain with evidence of elevation of cardiac enzymes and electrocardiogram changes to confirm myocardial infarction.

Diagnosis: synchronous AIS (a sudden onset of focal neurological deficit caused by an acute focal injury to the central nervous system due to a vascular narrowing cause) and AMI (acute elevation cardiac enzyme plus ischemic electrocardiogram and/or symptoms).

${ }^{\star}$ Correspondence to: Mohammed Habib, Cardiology Department- Al Shifa Hospital, Gaza, Palestine, E-mail: cardiomohammad@yahoo.com

Key words: cardio-cerebral infarction syndrome (ccis), diagnosis and treatment

Received: February 08, 2021; Accepted: February 19, 2021; Published: February 26, 2021 


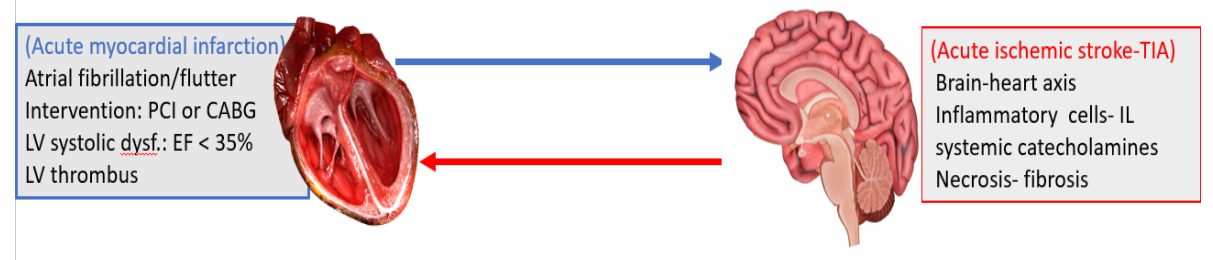

Figure 1. Definition of cardio-cerebral infarction syndrome

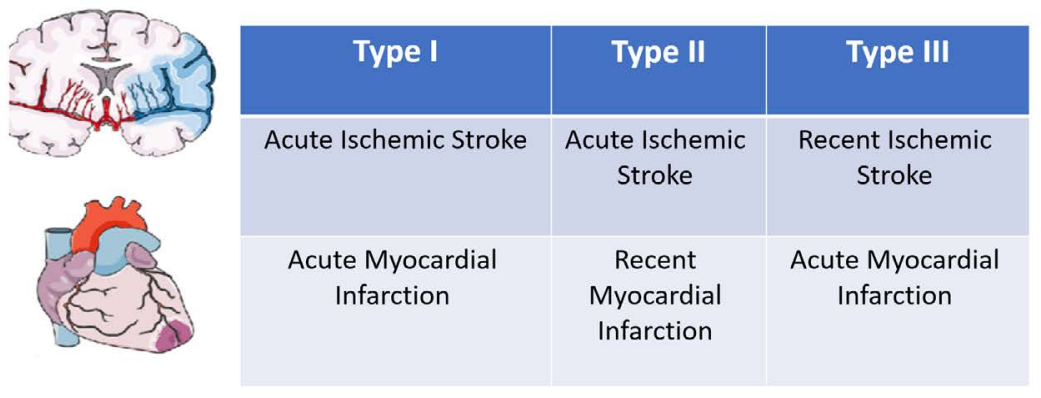

Figure 2. Types of cardio-cerebral infarction syndrome

Pathophysiology: the pathophysiology of simultaneous cardiocerebral infarction can be classified into three categories:

1. Cardiac conditions Type 1A: There are several conditions that lead to simultaneous acute cerebral and coronary infarction. The most of these is atrial fibrillation has been reported as a cause of simultaneous cardio-cerebral infarction due to common source of both cerebral and coronary embolism [9]. Type-I acute aortic dissection with dissection flap extending to coronary and common carotid arteries origin had been reported to cause concurrent acute myocardial infarction and acute ischemic stroke [10]. In addition, concurrent coronary and cerebral vasospasm due to electrical injury have been reported as an uncommon cause of simultaneous cardio-cerebral infarction [11] (Figure 3). Pre-existing intracardiac thrombus from left ventricular tumour or prosthetic valve thrombosis or impaired left ventricular ejection fraction can also lead to simultaneous coronary and cerebral vascular occlusion [12]. The thrombus formed in the right ventricle in acute right ventricular infarction with right ventricular dysfunction in combination with patent foramen ovale can embolize to both vascular territories. Severe hypotension or cardiogenic shock following AMI can also lead to hemodynamic stroke [13].

2. Brain causes (Brain-heart axis) Type 1B: Brain-heart axis dysregulation might be an alternative pathophysiology of simultaneous cardio-cerebral infarction syndrome. It has been shown that the insular cortex plays a critical role in central autonomic system regulation [14]. Patients with AIS in the parietoinsular region were found to have higher risk of developing arrythmias such as atrial fibrillation [15]. An abnormal electrocardiogram, including ST-segment elevation myocardial infarction, was found to be related to ischemic stroke in the insular cortex [16]. In addition to electrocardiographic abnormalities, myocardial damage determined by elevated serum cardiac troponin $\mathrm{T}$ was shown to be associated with acute cerebral infarction in specific brain regions including the right insular and right inferior parietal lobule [17]. Cardiac sympathetic overactivity from an insular cortex lesion can provoke diffuse myocardial damage, "myocytolysis," which leads to elevation of cardiac enzyme [18]. Results from human studies showed that the stimulation of different sides of the insular cortex resulted to different cardiac autonomic responses. And the right-side stimulation of insular cortex resulted in a predominant sympathetic effect, whereas the left-side stimulation resulted in a predominant parasympathetic effect [18].

3. Non cardiac and non-brain causes: Type 1C: Coronavirus disease 2019 (COVID-19) infection and Type I cardio-cerebral infarction syndrome: Recent studies suggested that coronavirus disease 2019 (COVID-19) infection can be increased the risk of AIS and AMI. However, the evidence base is limited mainly to case reports and 2 cohort studies. The evidence that COVID-19 may increase the risk of acute ischemic cardiovascular events. the underlying mechanisms may cytokine-mediated hypercoagulability and plaque destabilization [19]. Severe hypotension can be causes infarction in brain and myocardial infarction.

\section{Treatment}

According to the scientific statement from the American Heart Association/American Stroke Association (AHA/ASA) guideline 2018, For patients presenting with concurrent AIS and acute MI, treatment with IV alteplase at the dose appropriate for cerebral ischemia, followed by percutaneous coronary intervention (PCI) and stenting if indicated, is reasonable [20], but no specific recommendation for patient with non-STEMI or for patient with contraindication for thrombolytic in STEMI patient.

\section{We recommended that:}

- If patient with STEMI and no contraindicated to thrombolytic treatment and hemodynamic stable, we recommended IV alteplase at the dose appropriate for cerebral ischemia then pharmacoinvasive PCI for STEMI.

- In patients with non-STEMI patients. Early invasive PCI and if the stroke related to large vessel occlusion mechanical thrombectomy is recommended.

- If patient contraindicated to thrombolytic treatment and hemodynamic instability, we recommended primary PCI for STEMI patients and early invasive strategy for non-STEMI patients. If the stroke related to large vessel occlusion mechanical thrombectomy is recommended (Figure 4). 
- Atrial fibrillation has been reported as a cause of simultaneous cardio-cerebral infarction due to common source of both cerebral and coronary embolism

- Type-I acute aortic dissection with dissection flap extending to coronary and common carotid arteries origin had been reported to cause concurrent acute myocardial infarction and acute ischemic stroke

- In addition, concurrent coronary and cerebral vasospasm due to electrical injury have been reported as an uncommon cause of simultaneous cardio-cerebral infarction

- Pre-existing intracardiac thrombus from poor left ventricular ejection fraction can also lead to simultaneous coronary and cerebral vascular occlusion

- Likewise, a thrombus formed in the right ventricle in acute right ventricular infarction with right ventricular failure in combination with patent foramen ovale can embolize to both vascular territories

- Severe hypotension following acute myocardial infarction can also lead to hemodynamic stroke

- prosthetic valve thrombosis

- Intracardiac masses (myxoma- papillary fibroelastoma)

- Infective endocarditis

Figure 3. Cardiac conditions cause type 1 cardio-cerebral infarction syndrome

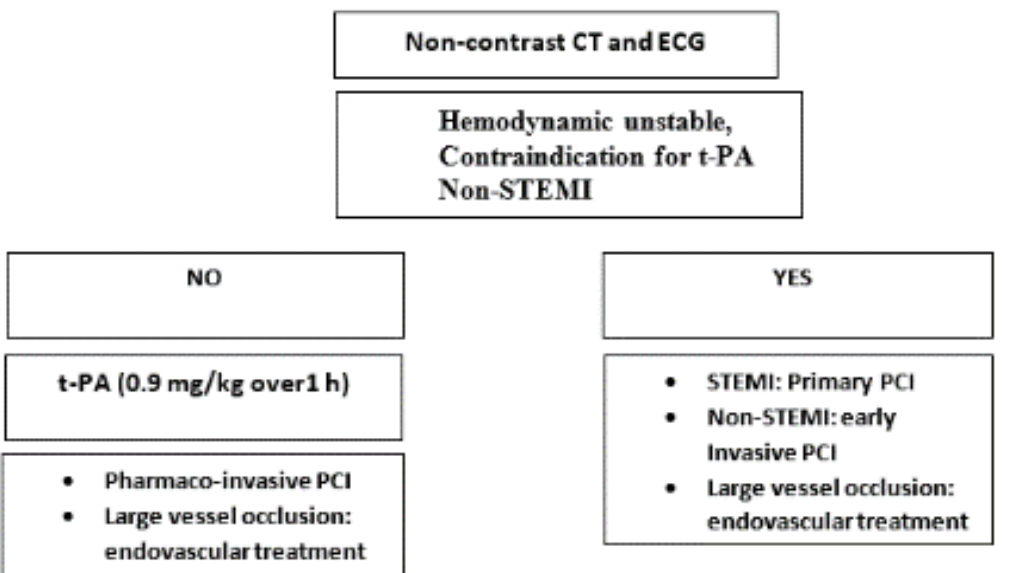

Figure 4. Treatment of type 1 cardio-cerebral infarction syndrome

PCI: percutaneous coronary intervention, STEMI: ST elevation myocardial infarction, Non-STEMI: non-STE elevation myocardial infarction

\section{Antiplatelet/anticoagulation treatment}

(Triple 1 week, Dual 6 months, then single OAC or aspirin lifelong)

Triple 1 week: Aspirin (75-100 mg) + Clopidogrel (75 mg) + OAC (Rivaroxaban $2.5 \mathrm{mg}$ twice or warfarin: INR $2-3$ and TTR $>70 \%$ )

Dual: (N)OAC + antiplatelet (1 week-6 months)

AF: Clopidogrel+ OAC (Clopidogrel (75 mg) + OAC (Rivaroxaban $15 \mathrm{mg}$ OD (GFR <60: $10 \mathrm{mg}$ ) or warfarin: INR 2-3 and TTR > 70\%)

LVT: first 3 months: Clopidogrel + OAC (Clopidogrel (75 mg) + OAC (Rivaroxaban $15 \mathrm{mg}$ OD (GFR <60: $10 \mathrm{mg}$ ) or warfarin: INR 2-3 and TTR > 70\%). Last 3 months: Aspirin (75-100 mg) + Clopidogrel (75 mg)

\section{Single After 6 months: (N)OAC or antiplatelet}

AF: Rivaroxaban or warfarin (Rivaroxaban $20 \mathrm{mg}$ OD (GFR <60: $15 \mathrm{mg}$ ) or warfarin: INR 2-3 and TTR > 70\%), LVT: aspirin $100 \mathrm{mg}$ tab one daily.

Type 2: acute ischemic stroke after recent myocardial infarction
Definition: Acute ischemic stroke in patients with history of Myocardial infarction in the previous 3 months.

Diagnosis: AIS (a sudden onset of focal neurological deficit caused by an acute focal injury to the central nervous system due to a vascular narrowing cause)) and history of AMI (acute elevation cardiac enzyme plus ischemic electrocardiogram and/or symptoms) in the previous 3 months.

Pathophysiology (Figure 4):

1. left ventricular mural thrombus (LVMT) due to impaired left ventricle ejection fraction $(\mathrm{EF})<35 \%$ and regional wall motion abnormalities such as dyskinesia or akinesia and septi-apical wall role the most important risk factor. The LVMT is most likely to occur by 2 weeks after an MI in $0.6-3.7 \%$ of patients [21]. new pharmacological therapy with dual antiplatelet agents and antithrombotic agents along with early revascularization procedures, might have contributed to the reduction in LVMT formation after myocardial infarction [22]. Increased coagulation activity during AMI, can potentially lead to increased thrombosis and subsequent thromboembolic events including stroke. 
2. The circulatory inflammatory cytokines may be initiated a cascade of events in the cerebral circulation. this phenomenon may contribute to plaque rupture and subsequent thrombus formation in the cerebral circulation [23].

3. Revascularization with early percutaneous coronary intervention (PCI) has become the standard of care for patients with acute myocardial infarction and coronary artery bypass graft surgery (CABG) were associated with increased stroke risk. Similarly, analysis of the OASIS [24] registry found that patients with higher rates of invasive cardiac procedures (CABG and PCI) suffered from increased risk of ischemic stroke at 6 months $(\mathrm{p}=0.004)$.

4. Atrial fibrillation and atrial flutter after myocardial infarction increased risk of ischemic stroke and occurs in up to $20 \%$ of patients and can cause increased in-hospital and long-term mortality [25].

\section{Treatment:}

According to the scientific statement from the American Heart (Figure 5).

Association/American Stroke Association (AHA/ASA) guideline 2018, [20]

1. For patients presenting with AIS and a history of recent MI in the past $3 \mathrm{mo}$, treating the ischemic stroke with IV alteplase is reasonable if the recent MI was non-STEMI (Class IIa).

2. For patients presenting with AIS and a history of recent MI in the past $3 \mathrm{mo}$, treating the ischemic stroke with IV alteplase is reasonable if the recent MI was a STEMI involving the right or inferior myocardium (Class IIa).

3. For patients presenting with AIS and a history of recent MI in the past 3 mo, treating the ischemic stroke with IV alteplase may reasonable if the recent MI was a STEMI involving the left anterior myocardium (Class IIb).

The safety of IV r-tPA (intravenous tissue-type plasminogen activator) for acute ischemic stroke (AIS) treatment after recent myocardial infarction (MI) is still a matter of debate. In recent Retrospective review of consecutive 102 AIS patients admitted for AIS with history of MI in the previous 3 months. Patients were divided into 2 groups: treated or not treated with standard IV r-tPA dose for AIS. Four (8.5\%) IV r-tPA-treated patients died from confirmed or presumed cardiac rupture/ tamponade, all with a STEMI in the week preceding stroke. This complication occurred in $1(1.8 \%)$ patient in the nontreated group $(\mathrm{P}=0.178)$. and no non-STEMI patients receiving IV r-tPA had cardiac complications [26]. Beyond bleeding complications, the main concerns about giving rtPA to patients with a history of recent MI are [1] the potential for thrombolysis-induced myocardial haemorrhage predisposing to myocardial wall rupture, [2] postmyocardial infarction pericarditis that may become hemopericardium, and [3] possible ventricular thrombi that could embolize because of thrombolysis.

\section{1- Left ventricle thrombus formation \\ 2- Increased coagulation activity \\ 3- The circulatory inflammatory cytokines \\ 4- Post myocardial infarction atrial fibrillation/ atrial flutter \\ 5- Intervention of myocardial infarction ( $\mathrm{PCl}$ and $\mathrm{CABG}$ )}

Figure 5. Causes of acute ischemic stroke after myocardial infarction
The new recommendation according to European Stroke Organisation (ESO) 2021 guidelines on intravenous thrombolysis for acute ischemic stroke Recommendation [27]:

- Contraindication of t-PA For patients with acute ischemic stroke of $<4.5 \mathrm{~h}$ duration and with history of subacute $(>6 \mathrm{~h})$ ST elevation myocardial infarction during the last seven days.

- For patients with acute ischemic stroke of $<4.5 \mathrm{~h}$ duration and with history of ST-elevation myocardial infarction of more than a week to three months, there is insufficient evidence to make a recommendation.

- For patients with acute ischemic stroke of $<4.5 \mathrm{~h}$ duration and with a history of non-ST-elevation myocardial infarction during the last three months, we suggest intravenous thrombolysis with alteplase.

The recent trial suggested that Among 40396 AIS patients aged $\geq 65$ years treated with $\mathrm{rtPA}, 241(0.6 \%)$ had recent $\mathrm{MI}$ in the past 3 months, of which $19.5 \%$ (41 patients) were ST-segment-elevation myocardial infarction. Patients with recent MI had more severe stroke than those without. Among older patients receiving rtPA for AIS, a recent history of $\mathrm{MI}$ in the past 3 months was associated with higher inhospital mortality compared with no history of MI in ischemic stroke patients treated with rtPA and the association was more prominent in patients with STEMI than those with NSTEMI. This association was not significant, if the time frame from the onset of MI to the indexed AIS was $>3$ months. Despite the increasing risk, further studies are needed to evaluate the benefit of rtPA, the only approved medical therapy, in AIS patients with recent MI [28]. Further investigations are necessary to determine whether the benefit of recombinant tissue-type plasminogen activator outweighs its risk among AIS patients with a recent history of MI.

At final we recommended that the treatment of type II cardiocerebral infarction (Figure 6):

1. For patients with acute ischemic stroke of $<4.5 \mathrm{~h}$ duration and with a history of non-ST-elevation myocardial infarction during the last three months, we suggest intravenous thrombolysis with alteplase.

2. Mechanical thrombectomy may be a therapeutic alternative in patients with large vessel occlusion and recent STEMI.

3. Anticoagulation with rivaroxaban and clopidogrel is recommended in patients with AIS related to cardioembolic causes (left ventricle thrombus and/or atrial fibrillation) for at least 3 months for left ventricle thrombus and 3 months rivaroxaban and clopidogrel then rivaroxaban lifelong for atrial fibrillation [29].

\section{Antiplatelet/anticoagulation treatment:}

According to time from myocardial infarction (Triple 1 week, Dual 6 months, then single OAC or aspirin lifelong)

- Triple 1 week: Aspirin (75-100 mg) + Clopidogrel (75 mg) + OAC (Rivaroxaban $2.5 \mathrm{mg}$ twice or warfarin: INR 2-3 and TTR > 70\%)

\section{- Dual: (N)OAC + antiplatelet (1 week-6 months):}

AF: Clopidogrel+ OAC (Clopidogrel (75 mg) + OAC (Rivaroxaban $15 \mathrm{mg}$ OD (GFR <60: $10 \mathrm{mg}$ ) or warfarin: INR 2-3 and TTR > 70\%)

LVT: first 3 months: Clopidogrel+ OAC (Clopidogrel $(75 \mathrm{mg})+$ OAC (Rivaroxaban $15 \mathrm{mg}$ OD (GFR <60: $10 \mathrm{mg}$ ) or warfarin: INR 2-3 and TTR > 70\%). Last 3 months: Aspirin (75-100 mg) + Clopidogrel (75 mg) 


\section{- Single After 6 months: (N)OAC or antiplatelet:}

AF: Rivaroxaban or warfarin (Rivaroxaban $20 \mathrm{mg}$ OD (GFR <60: $15 \mathrm{mg}$ ) or warfarin: INR 2-3 and TTR > 70\%), LVT: aspirin $100 \mathrm{mg}$ tab once daily.

Type 3: Acute myocardial infarction after recent ischemic stroke:

Definition: AMI in patients with history of AIS in the previous 3 months.

Diagnosis: AMI (acute elevation cardiac enzyme plus ischemic electrocardiogram and/or symptoms) and history of AIS (a sudden onset of focal neurological deficit caused by an acute focal injury to the central nervous system due to a vascular narrowing cause) in the previous 3 months

\section{Pathophysiology:}

In general, the risk of acute myocardial infarction after ischemic stroke was low. The 5-year cumulative incidence of acute myocardial infarction was $2 \%$, incidence of AMI was highest in the first year and especially during the first 2 months after AIS. Notably, patients with history of coronary heart disease showed a 5-fold risk of acute myocardial infarction after stroke onset, and those with cardioembolism subtype had a higher risk than other subtypes [7]. Also, poststroke cardiac arrhythmias could be another possible cause of AMI after AIS.

\section{Treatment}

Revascularization: the use of thrombolytics is contraindicated and primary PCI for STEMI and early invasive PCI strategy for non-STEMI patient is recommended (Figure 7).
Anticoagulation and antiplatelet for Cardio-embolic Causes $[29,30]:$

Antiplatelet/anticoagulation treatment:

(Triple 1 week, Dual 6 months, then single OAC or aspirin lifelong)

- Triple 1 week: Aspirin (75-100 mg) + Clopidogrel (75 mg) + OAC (Rivaroxaban $2.5 \mathrm{mg}$ twice or warfarin: INR 2-3 and TTR > 70\%)

- Dual: (N)OAC + antiplatelet (1 week-6 months):

AF: Clopidogrel+ OAC (Clopidogrel (75 mg) + OAC (Rivaroxaban $15 \mathrm{mg}$ OD (GFR <60: $10 \mathrm{mg}$ ) or warfarin: INR 2-3 and TTR > 70\%)

LVT: first 3 months: Clopidogrel+ OAC (Clopidogrel $(75 \mathrm{mg})+$ OAC (Rivaroxaban $15 \mathrm{mg}$ OD (GFR <60: $10 \mathrm{mg}$ ) or warfarin: INR 2-3 and TTR > 70\%). Last 3 months: Aspirin (75-100 mg) + Clopidogrel (75 mg)

- Single After 6 months: (N)OAC or antiplatelet:

AF: Rivaroxaban or warfarin (Rivaroxaban $20 \mathrm{mg}$ OD (GFR <60: $15 \mathrm{mg}$ ) or warfarin: INR 2-3 and TTR > 70\%), LVT: aspirin $100 \mathrm{mg}$ tab once daily.

\section{Conclusion}

In type 1 cardio-cerebral infarction: For patients presenting with concurrent AIS and acute MI, treatment with IV alteplase at the dose appropriate for cerebral ischemia, followed by percutaneous coronary intervention (PCI) and stenting if indicated. But if patient contraindicated to thrombolytic treatment and/or hemodynamic instability we recommended primary PCI for STEMI patients and early invasive strategy for non-STEMI patients. And if the stroke related to large vessel occlusion mechanical thrombectomy is recommended.

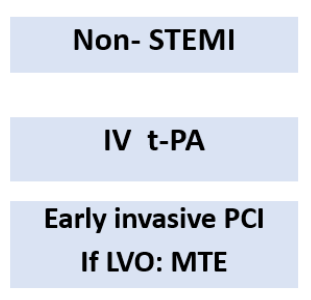

Figure 6. Summary of treatment for type II

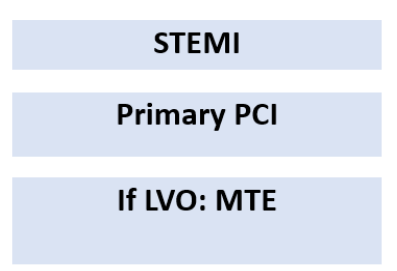

\section{Acute myocardial infarction and history of ischemic stroke within previous 3 months}

- PPCI for STEMI

- Early Invasive PCI for Non-STEMI 
In type II cardio-cerebral infarction: For patients with acute ischemic stroke of $<4.5 \mathrm{~h}$ duration and with a history of recent nonST-elevation myocardial infarction during the last three months, we suggest intravenous thrombolysis with alteplase, and Mechanical thrombectomy may be a therapeutic alternative in patients with large vessel occlusion and recent STEMI.

In type III cardio-cerebral infarction: the use of thrombolytics is contraindicated and primary PCI for STEMI and early invasive PCI strategy for non-STEMI patient is recommended.

\section{References}

1. Al Suwaidi J, Al Habib K, Asaad N, Singh R, Hersi A, et al. (2012) Immediate and one-year outcome of patients presenting with acute coronary syndrome complicated by stroke: findings from the 2nd Gulf Registry of Acute Coronary Events (Gulf RACE-2). BMC Cardiovasc Disord 12: 64. [Crossref]

2. Longstreth WT Jr, Litwin PE, Weaver WD (1993) Myocardial infarction, thrombolytic therapy, and stroke. A community-based study. The MITI Project Group. Stroke 24: 587-590. [Crossref]

3. Kajermo U, Ulvenstam A, Modica A, Jernberg T, Mooe T (2014) Incidence, trends, and predictors of ischemic stroke 30 days after an acute myocardial infarction. Stroke 45 : 1324-1330. [Crossref]

4. Mooe T, Olofsson BO, Stegmayr B, Eriksson P (1999) Ischemic stroke. Impact of a recent myocardial infarction. Stroke 30: 997-1001. [Crossref]

5. Brammås A, Jakobsson S, Ulvenstam A, Mooe T (2013) Mortality after ischemic stroke in patients with acute myocardial infarction: predictors and trends over time in Sweden. Stroke 44: 3050-3055. [Crossref]

6. Witt BJ, Ballman KV, Brown Jr RD, Meverden RA, Jacobsen SJ, et al. (2006) The Incidence of Stroke after Myocardial Infarction: A Meta-Analysis. Am J Med 119: 354. e1-354. [Crossref]

7. Lee K, Kim S, Kim JP, Kang J, Kim BJ, et al. (2021) Five-Year Risk of Acute Myocardial Infarction After Acute Ischemic Stroke in Korea. J Am Heart Assoc 10: e018807. [Crossref]

8. Yeo LLL, Andersson T, Yee KW, Tan BYQ, Paliwal P, et al. (2017) Synchronous cardiocerebral infarction in the era of endovascular therapy: which to treat first? $J$ Thromb Thrombolysis 44: 104-111. [Crossref]

9. Tokuda K, Shindo S, Yamada K, Shirakawa M, Uchida K, et al. (2016) Acute embolic cerebral infarction and coronary artery embolism in a patient with atrial fibrillation caused by similar thrombi. J Stroke Cerebrovasc Dis 25: 1797-1799. [Crossref]

10. Nguyen TL, Rajaratnam R (2011) Dissecting out the cause: a case of concurrent acute myocardial infarction and stroke. BMJ Case Rep 2011: bcr0220113824. [Crossref]

11. Verma GC, Jain G, Wahid A, Saurabh C, Sharma NK, et al. (2014) Acute ischaemic stroke and acute myocardial infarction occurring together in domestic low-voltage (220-240V) electrical injury: a rare complication. J Assoc Physicians India 62: 620623. [Crossref]

12. Yeo LL, Andersson T, Yee KW, Tan BY, Paliwal P, et al. (2017) Synchronous cardiocerebral infarction in the era of endovascular therapy: which to treat first? $J$ Thromb Thrombolysis 44: 104-111. [Crossref]

13. Omar HR, Fathy A, Rashad R, Helal E (2010) Concomitant acute right ventricular infarction and ischemic cerebrovascular stroke; possible explanations. Int Arch Med 3: 25. [Crossref]
14. Nagai M, Hoshide S, Kario K (2010) The insular cortex and cardiovascular system: a new insight into the brain-heart axis. J Am Soc Hypertens 4: 174-182. [Crossref]

15. Vingerhoets F, Bogousslavsky J, Regli F, Van Melle G (1993) Atrial fibrillation after acute stroke. Stroke 24: 26-30. [Crossref]

16. Christensen H, Boysen G, Christensen AF, Johannesen HH (2005) Insular lesions, ECG abnormalities, and outcome in acute stroke. J Neurol Neurosurg Psychiatry 76: 269271. [Crossref]

17. Ay H, Koroshetz WJ, Benner T, Vangel MG, Melinosky C, et al. (2006) Neuroanatomic correlates of stroke-related myocardial injury. Neurology 66: 1325-1329. [Crossref]

18. Cheshire WP Jr, Saper CB (2006) The insular cortex and cardiac response to stroke. Neurology 66: 1296-1297. [Crossref]

19. Modin D, Claggett B, Sindet-Pedersen C, Lassen MCH, Skaarup KG, et al. (2020) Acute COVID-19 and the Incidence of Ischemic Stroke and Acute Myocardial Infarction. Circulation 142: 2080-2082. [Crossref]

20. Powers WJ, Rabinstein AA, Ackerson T, Adeoye OM, Bambakidis NC, et al. (2018) 2018 Guidelines for the early management of patients with acute ischemic stroke: a guideline for healthcare professionals from the American Heart Association/American Stroke Association. Stroke 49: e46-e110. [Crossref]

21. Greaves SC, Zhi G, Lee RT, Solomon SD, MacFadyen J, et al. (1997) Incidence and natural history of left ventricular thrombus following anterior wall acute myocardial infarction. Am J Cardiol 80: 442-448. [Crossref]

22. Motro M, Barbash GI, Hod H, Kaplinsky E, Laniado S, et al (1991) Incidence of left ventricular thrombi formation after thrombolytic therapy with recombinant tissue plasminogen activator, heparin, and aspirin in patients with acute myocardial infarction. Am Heart J 122: 23-26. [Crossref]

23. Lombardo A, Biasucci LM, Lanza GA, Coli S, Silvestri P, et al. (2004) inflammation possible link between coronary and carotid plaque instability. Circulation 109: 31583163. [Crossref]

24. Yusuf S, Flather M, Pogue J, Hunt D, Varigos J, et al. (1998) Variations between countries in invasive cardiac proce-dures and outcomes in patients with suspected unstable angina or myocardial infarction without initial ST elevation. OASIS Registry investigators. Lancet 352: 507-514. [Crossref]

25. Healey JS, Connolly SJ, Gold MR, Israel CW, Van Gelder IC, et al. (2012) Subclinical atrial fibrillation and the risk of stroke. $N$ Engl J Med 366: 120-129. [Crossref]

26. Marto JP, Kauppila LA, Jorge C, Calado S, Viana-Baptista M, et al. (2019) Intravenous Thrombolysis for Acute Ischemic Stroke After Recent Myocardial Infarction Case Series and Systematic Review. Stroke 50: 2813-2818. [Crossref]

27. Berge E, Whiteley W, Audebert H, De Marchis GM, Fonseca AC, et al. (2021) European Stroke Organisation (ESO) guidelines on intravenous thrombolysis for acute ischaemic stroke. Eur Stroke J 6: I-LXII. [Crossref]

28. Inohara T, Liang L, Kosinski AS, Smith EE, Schwamm LH, et al. (2019) Recent Myocardial Infarction is Associated with Increased Risk in Older Adults with Acute Ischemic Stroke Receiving Thrombolytic Therapy. J Am Heart Assoc 8: e012450. [Crossref]

29. Collet J, Thiele H, Barbato E, Barthélémy O, Bauersachs J, et al. (2021) 2020 Acute Coronary Syndromes (ACS) in Patients Presenting without Persistent ST-Segment Elevation (Management of) Guidelines. Eur Heart J 42: 1289-1367. [Crossref]

30. Gibson CM, Mehran R, Bode C, Halperin J, Verheugt FW, et al. (2016) Prevention of Bleeding in Patients with Atrial Fibrillation Undergoing PCI. N Engl J Med 375 : 2423-2434. [Crossref]

Copyright: (C2021 Habib M. This is an open-access article distributed under the terms of the Creative Commons Attribution License, which permits unrestricted use, distribution, and reproduction in any medium, provided the original author and source are credited. 